\title{
The Scientific Importance of Returning Airfall Dust as a Part of Mars Sample Return (MSR)
}

\author{
Monica M. Grady, Roger E. Summons, ${ }^{2}$ Timothy D. Swindle, ${ }^{3}$ Frances Westall, ${ }^{4}$ Gerhard Kminek, ${ }^{5}$ \\ Michael A. Meyer, ${ }^{6}$ David W. Beaty, ${ }^{7}$ Brandi L. Carrier, ${ }^{7}$ Timothy Haltigin, ${ }^{8}$ Lindsay E. Hays, ${ }^{6}$ Carl B. Agee, ${ }^{9}$ \\ Henner Busemann, ${ }^{10}$ Barbara Cavalazzi, ${ }^{11}$ Charles S. Cockell, ${ }^{12}$ Vinciane Debaille, ${ }^{13}$ Daniel P. Glavin, ${ }^{14}$ \\ Ernst Hauber, ${ }^{15}$ Aurore Hutzler, ${ }^{5}$ Bernard Marty, ${ }^{16}$ Francis M. McCubbin, ${ }^{17}$ Lisa M. Pratt, ${ }^{18}$ \\ Aaron B. Regberg, ${ }^{17}$ Alvin L. Smith, ${ }^{7}$ Caroline L. Smith, ${ }^{19,20}$ Kimberly T. Tait, ${ }^{21}$ Nicholas J. Tosca, ${ }^{22}$ \\ Arya Udry, ${ }^{23}$ Tomohiro Usui, ${ }^{24}$ Michael A. Velbel, ${ }^{25,26}$ Meenakshi Wadhwa, ${ }^{7,27}$ and Maria-Paz Zorzano ${ }^{28,29}$
}

\footnotetext{
${ }^{1}$ The Open University, Milton Keynes, UK.

${ }^{2}$ Massachusetts Institute of Technology, Earth, Atmospheric and Planetary Sciences, Cambridge, Massachusetts, USA.

${ }^{3}$ University of Arizona, Lunar and Planetary Laboratory, Tucson, Arizona, USA.

${ }^{4}$ Centre National de la Recherche Scientifique (CNRS), Centre de Biophysique Moléculaire, Orléans, France.

${ }^{5}$ European Space Agency, Noordwijk, The Netherlands.

${ }^{6}$ NASA Headquarters, Mars Sample Return Program, Washington, DC, USA.

${ }^{7}$ Jet Propulsion Laboratory, California Institute of Technology, Pasadena, California, USA.

${ }^{8}$ Canadian Space Agency, Saint-Hubert, Quebec, Canada.

${ }^{9}$ University of New Mexico, Institute of Meteoritics, Albuquerque, New Mexico, USA.

${ }^{10}$ ETH Zürich, Institute of Geochemistry and Petrology, Zürich, Switzerland.

${ }^{11}$ Università di Bologna, Dipartimento di Scienze Biologiche, Geologiche e Ambientali, Bologna, Italy.

${ }^{12}$ University of Edinburgh, Centre for Astrobiology, School of Physics and Astronomy, Edinburgh, UK.

${ }^{13}$ Université Libre de Bruxelles, Bruxelles, Belgium.

${ }_{15}^{14}$ NASA Goddard Space Flight Center, Solar System Exploration Division, Greenbelt, Maryland, USA.

${ }^{15}$ German Aerospace Center (DLR), Institute of Planetary Research, Berlin, Germany.

${ }^{16}$ Université de Lorraine, CNRS, CRPG, Nancy, France.

${ }^{17}$ NASA Johnson Space Center, Astromaterials Research and Exploration Science Division, Houston, Texas, USA.

${ }^{18}$ Indiana University Bloomington, Earth and Atmospheric Sciences, Bloomington, Indiana, USA.

${ }^{19}$ Natural History Museum, Department of Earth Sciences, London, UK.

${ }^{20}$ University of Glasgow, School of Geographical and Earth Sciences, Glasgow, UK.

${ }_{22}^{21}$ Royal Ontario Museum, Department of Natural History, Toronto, Ontario, Canada.

${ }^{22}$ University of Cambridge, Department of Earth Sciences, Cambridge, UK.

${ }^{23}$ University of Nevada Las Vegas, Las Vegas, Nevada, USA.

${ }^{24}$ Japan Aerospace Exploration Agency (JAXA), Institute of Space and Astronautical Science (ISAS), Chofu, Tokyo, Japan.

${ }^{25}$ Michigan State University, Earth and Environmental Sciences, East Lansing, Michigan, USA.

${ }^{26}$ Smithsonian Institution, Department of Mineral Sciences, National Museum of Natural History, Washington, DC, USA.

${ }^{27}$ Arizona State University, Tempe, Arizona, USA.

${ }^{28}$ Centro de Astrobiologia (CSIC-INTA), Torrejon de Ardoz, Spain.

${ }^{29}$ University of Aberdeen, Department of Planetary Sciences, School of Geosciences, King's College, Aberdeen, UK.
}

This paper was written by the MSR Science Planning Group 2 (MSPG2) working under Terms of Reference from NASA and ESA.

(C) Monica M. Grady et al., 2021; Published by Mary Ann Liebert, Inc. This Open Access article is distributed under the terms of the Creative Commons License (http://creativecommons.org/licenses/by/4.0), which permits unrestricted use, distribution, and reproduction in any medium, provided the original work is properly cited. 


\section{Table of Contents}

Abstract

Summary of Findings

1. Introduction

1.1. Previous dust studies

1.2. The martian dust cycle

2. What Could be Learned from Samples of Martian Dust?

2.1. Chemical, mineralogical and isotopic studies

2.2. Study of carbon chemistry

2.3. Corrosion

2.4. Importance of physical and magnetic properties

2.5. Atmospheric dynamics and global circulation

2.6. Need for returned samples

3. How Much Dust is Required?

3.1. How much airfall dust might we collect?

3.2. Airborne dust-a potential additional sampling opportunity

4. Summary

Acknowledgments

Disclosure Statement

Funding Information

Acronyms Used

References

\section{Abstract}

Dust transported in the martian atmosphere is of intrinsic scientific interest and has relevance for the planning of human missions in the future. The MSR Campaign, as currently designed, presents an important opportunity to return serendipitous, airfall dust. The tubes containing samples collected by the Perseverance rover would be placed in cache depots on the martian surface perhaps as early as 2023-24 for recovery by a subsequent mission no earlier than 2028-29, and possibly as late as 2030-31. Thus, the sample tube surfaces could passively collect dust for multiple years. This dust is deemed to be exceptionally valuable as it would inform our knowledge and understanding of Mars' global mineralogy, surface processes, surface-atmosphere interactions, and atmospheric circulation. Preliminary calculations suggest that the total mass of such dust on a full set of tubes could be as much as $100 \mathrm{mg}$ and, therefore, sufficient for many types of laboratory analyses. Two planning steps would optimize our ability to take advantage of this opportunity: (1) the dust-covered sample tubes should be loaded into the Orbiting Sample container (OS) with minimal cleaning and (2) the capability to recover this dust early in the workflow within an MSR Sample Receiving Facility (SRF) would need to be established. A further opportunity to advance dust/atmospheric science using MSR, depending upon the design of the MSR Campaign elements, may lie with direct sampling and the return of airborne dust.

\section{Summary of Findings}

FINDING D-1: An accumulation of airfall dust would be an unavoidable consequence of leaving M2020 sample tubes cached and exposed on the surface of Mars. Detailed laboratory analyses of this material would yield new knowledge concerning surface-atmosphere interactions that operate on a global scale, as well as provide input to planning for the future robotic and human exploration of Mars.

FINDING D-2: The detailed information that is possible from analysis of airfall dust can only be obtained by investigation in Earth laboratories, and thus this is an important corollary aspect of MSR. The same information cannot be obtained from orbit, from in situ analyses, or from analyses of samples drilled from single locations.

FINDING D-3: Given that at least some martian dust would be on the exterior surfaces of any sample tubes returned to Earth, the capability to receive and curate dust in an MSR Sample Receiving Facility (SRF) is essential.
SUMMARY STATEMENT: The fact that any sample tubes cached on the martian surface would accumulate some quantity of martian airfall dust presents a low-cost scientifically valuable opportunity. Some of this dust would inadvertently be knocked off as part of tube manipulation operations, but any dust possible should be loaded into the OS along with the sample tubes. This dust should be captured in an SRF and made available for detailed scientific analysis.

\section{Introduction}

D UST IS PERVASIVE on Mars, in the air and in the regolith. Planning of the current MSR Campaign has already taken into account the need and opportunity to carry out bulk sampling of granular materials from the martian regolith (see E2E-iSAG, 2011; M2020 SDT, 2014; iMOST, 2019; and references therein). The hardware necessary to do this, namely, the "waterfall" bit on M2020 and associated sample 
tubes, has already been designed, built, and sent to Mars (Moeller et al., 2021). The next step (as of this writing) would be for the M2020 science team to evaluate the properties of regolith within the area they are exploring and decide which, and how many, such samples to collect. In addition, the MSR Campaign would unavoidably return some quantity of what we are calling serendipitous airfall dust. This is dust that falls out of the martian atmosphere onto an exposed surface that could be returned to Earth. Any such dust would be additional returned sample mass and would be representive of a more global as opposed to a local sample.

The way the MSR Campaign is currently envisioned, there is a specific aspect that would enhance the quantity of airfall dust that could be returned. The M2020 sample-collecting rover is expected to acquire rock and regolith samples into its sample tubes, seal them, and then deposit some or all on the martian surface in cache depots for future retreival. The first sample tubes may be placed in a depot as early as 2023-24, and further tubes may be placed in that or other depots during the following years. Sample recovery operations by the Sample Fetch Rover (SFR) are currently planned not earlier than 2028-29, and possibly as late as 2030-31 (Meyer et al., 2021). Thus, at least some of the sample tubes would have multiple years on the martian surface to accumulate airfall dust. A key open question, therefore, is how valuable is this dust to science, and what, if anything, needs to be done to capitalize on this scientific opportunity?

\subsection{Previous dust studies}

Previous studies of dust on Earth, from the Moon, and on Mars underline its importance in understanding natural processes, although for very different reasons. On Earth, reflecting the dominance of its surface biology, particleborne bacteria and viruses have been detected above Earth's atmospheric Boundary Layer (Reche et al., 2018) (of variable height from $\sim 100 \mathrm{~m}$ above the ocean to $4000-5000 \mathrm{~m}$ above land). In this case, the materials characterized could have been derived from soil-dust or organic aggregates initially swept up in sea spray. There is no doubt about the existence of long-distance transport and survival of microbes on terrestrial atmospheric particulates (De Deckker et al., 2008). In the case of the Moon, for which mass is moved around the surface by ballistic processes, analyses of the shapes of the grains and their compositional and age distributions provided information on the global lunar impact flux (Zellner et al., 2009; Korotev et al., 2010) as well as regional compositional variation (Wagner, 2006; Zellner, 2016). The nature of the dust particles, for example, spherules or glass shards reflecting local or regional provenance, influences their measured isotopic ages $\left({ }^{40} \mathrm{Ar} /{ }^{39} \mathrm{Ar}\right)$ (Zellner and Delano, 2015; Zellner, 2016). These studies were performed on particles transported by impact processes, which can be larger than the dust that is transported through atmospheric processes, but they are illustrative of the potential scientific value of such samples.

Complementary to these scientific studies of lunar dust is a large body of work relating to the risk factors relevant to robotic and human exploration of the Moon (Wagner, 2006), much of which is related to the physical characteristics of the dust, such as composition, shape, size, specific gravity, relative density, cohesion, compressibility, shear strength, and thermal conductivity (Kring, 2006). The lunar missions catalogued numerous instances where dust caused problems including loss of surface visibility during descent of the lunar module. Dust contamination also negatively impacted the lander and command modules including on-board equipment such as cameras, watches, sampling equipment and the Lunar Rover. Particularly relevant to human exploration were the effects of dust on space suits and seals that caused difficulties in operating wrist and suit hose locks, abrasion of suit fabric leading to increased daily leak rates, helmet visor scratches that decreased visibility and problems of dust in zippers, etc.

On Mars, it has been known since the time the planet was only studied through telescopes that wind velocities are sufficient to lift dust high into the atmosphere where it could remain for months, affecting both atmospheric heat flow (Leovy et al., 1973) as well as temperature (Hanel et al., 1972). Differences in surface albedo were inferred to arise from size-sorting of dust particles (Sagan et al., 1971). At least one component was recognized as the clay mineral montmorillonite (Hunt et al., 1973), while other observations suggested that the topographic structure of the polar caps, the transport of volatiles between reservoirs, and the amount of dust in the atmosphere were coupled (Murray et al., 1972; Leovy, 1973). Continuing exploration by landed missions and orbiting spacecraft refined our understanding of dust distribution and its transportation as well as its composition (e.g., Edgett and Malin, 2000).

Most recently, a series of missions to the martian surface (Spirit, Opportunity, Curiosity, Phoenix, Perseverance, and Insight) have measured atmospheric dust opacities (the technical parameter "tau") and chemistries. These data can be compared with measurements made from orbit by orbiters, including Mars Global Surveyor (MGS), Odyssey, Mars Reconnaissance Orbiter (MRO) Mars Atmosphere and Volatile EvolutioN (MAVEN), and ExoMars Trace Gas Orbiter (TGO). Many of the observations by orbiting and landed spacecraft complement each other. For example, the discovery that $\mathrm{HCl}$ co-varies with dust abundance in Mars' atmosphere by TGO (Olsen et al., 2021) mirrors that of Curiosity at Gale Crater (Sutter et al., 2017), suggesting that dust is a vector for surface reaction products which then become distributed throughout the entire atmospheric column. The settling of dust out of the atmosphere on Mars happens at a rate that is very significant to spacecraft operations (see e.g., Fig. 1). In 2018, a dust storm over several months reduced the intensity of light received from the Sun by $97 \%$ resulting in the final shut-down of the Opportunity rover (Guzewich et al., 2019). Similarly, in 2020, the InSight lander began experiencing power problems due to dust loading of its solar panels (Lorenz et al., 2020). For a recent summary of the possible relationship of future human missions to Mars and dust in the martian atmosphere, please see the work of Levine et al. (2018).

\subsection{The martian dust cycle}

As summarized by the Mars Climate Modeling Center, dust is lifted from Mars' surface to the atmosphere through processes associated with the exchange of momentum and heat between the atmosphere and the surface (Kahre et al., 2018; https:/www.nasa.gov/ames/mcmc, 2019). Once dust leaves the surface, it is mixed vertically in the turbulent 

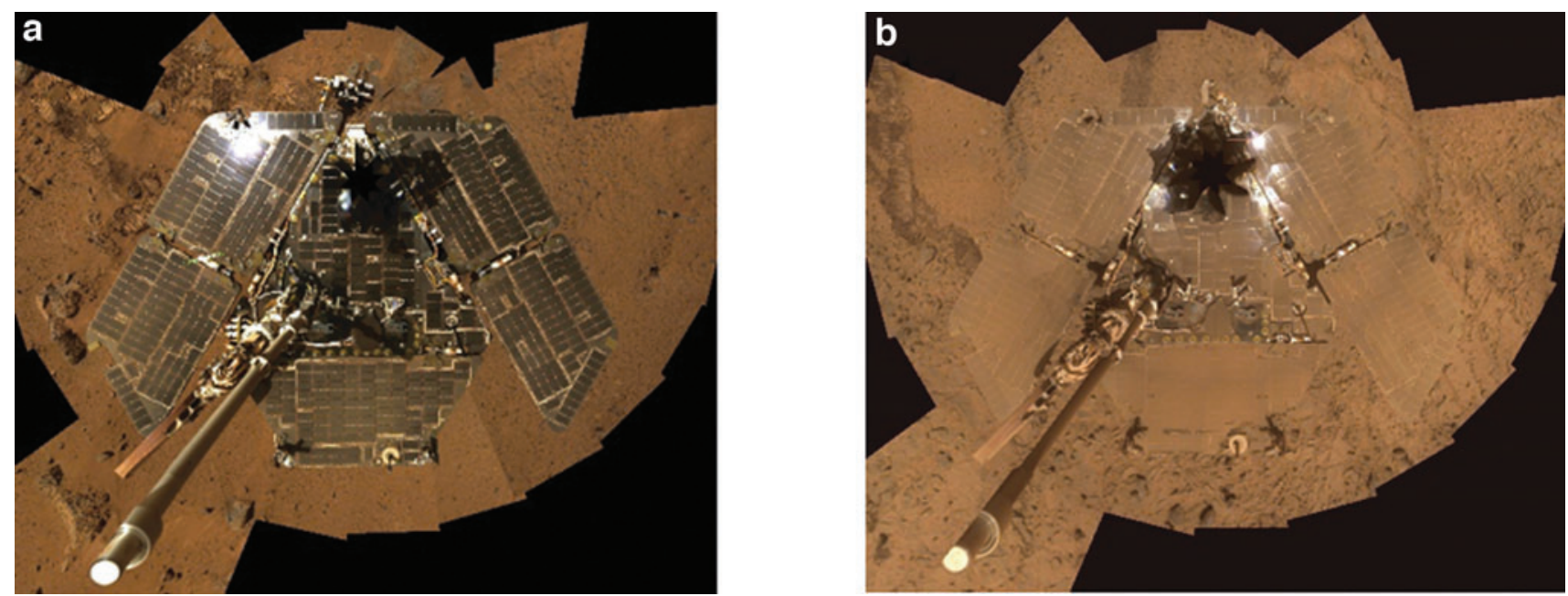

FIG. 1. Two images of the Spirit Exploration Rover taken by its Panoramic Camera (a) Sol 586; August 2005 (PIA 03272) (https://photojournal.jpl.nasa.gov/catalog/PIA03272); (b) Sol 1355-1358; October 2007 (PIA 10128) (https:// photojournal.jpl.nasa.gov/catalog/PIA10128). Note the accumulation of dust on the rover during the elapsed $2+$ years on the martian surface. If the same thing happens to the cached sample tubes, the dust on the outside of the tubes would be of significant scientific interest. (Image Credits: NASA/JPL-Caltech/Cornell)

planetary boundary layer and transported horizontally by large-scale winds before falling back to the surface though gravitation. This process has been happening on Mars for much of its geologic history. Thus, the martian dust cycle consists of repeated lifting, transportation, and deposition of grains. The places on Mars for which the dust lifting rates are higher than the deposition rates tend to be characterized by many bare outcrops, and where the reverse is true, there can be thick deposits of dust that have built up over time. While the dust is in the atmosphere, it is radiatively active and significantly impacts the atmospheric thermal structure. Warming and cooling modifiy pressure gradients, which in turn trigger winds and other kinds of atmospheric dynamics. Dust also plays a role in the height at which water condenses to form clouds during storms, contributing to the presence and distribution of water vapor in the upper atmosphere (Bertrand et al., 2020).

In this report, we draw an operational distinction between airborne dust (that which is in the atmosphere at the time of its sampling) and airfall dust (that which accumulates on a surface), recognizing that while the two categories of samples are geneticially related, collecting representative samples of each would require different approaches (see Section 4).

\section{What Could Be Learned from Samples of Martian Dust?}

Dust grains are composed of different minerals according to their origins and histories. The level of heterogeneity, particularly of isotopic effects, reflects the diversity of processes that occur across the entire planet. Such records of martian mineralogy and mineral chemistry have the potential to be informative about the redox states of redox-sensitive elements (e.g., $\mathrm{Cr}, \mathrm{Mn}$, and $\mathrm{Fe}$ ). Beyond the surface, dust also influences atmospheric chemistry and dynamics. Understanding the transport of dust to polar regions, and its accumulation there, is crucial to understanding the history of the polar caps. Dust samples would enable additional science because they are a proxy for planet-wide processes.

\subsection{Chemical, mineralogical and isotopic studies}

Orbiting spacecraft, landers, and rovers have measured the major and minor element compositions of airfall dust and established that there is a globally uniform component (see Figure 2). However, there have not been measurements of the elemental compositions of individual grains. Because the dust grains are so small, these are measurements that require instruments that are much more sensitive and sophisticated than can be deployed on Mars.

Bulk analyses by the CheMin instrument aboard the Curiosity rover revealed that up to $60 \%$ of some sediments in Gale Crater comprise an amorphous, non-crystalline component, representing mixtures of sedimentary materials that can only be estimated indirectly and which cannot be further characterized by XRD analysis alone (Smith et al.,

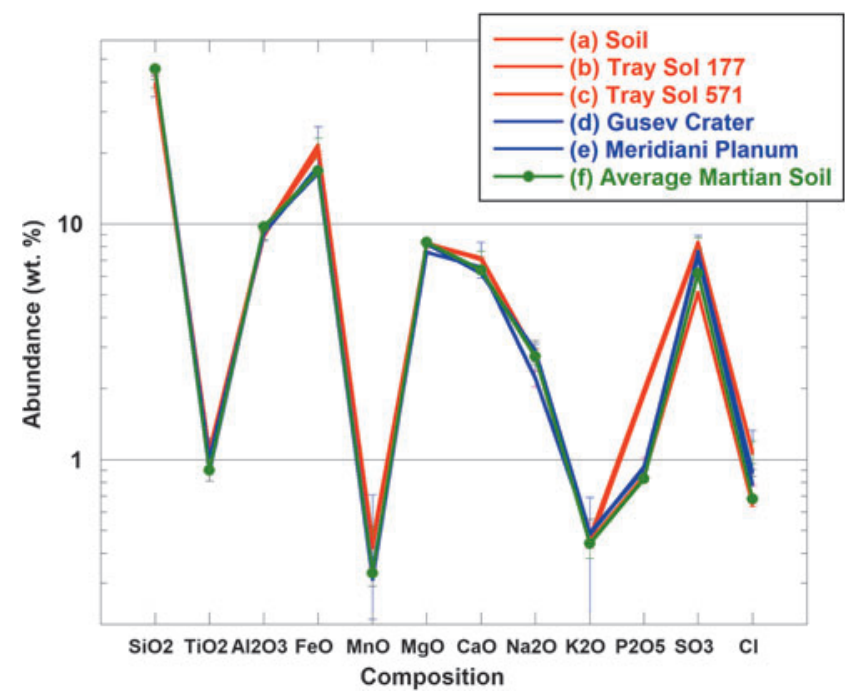

FIG. 2. The composition of soil from different locations across Mars. Locations (a)-(c) are from Gale Crater. Data compilation from Berger et al. (2016). 
2021). This component may comprise a mix of the regolith and airfall dust and may even host biosignatures. These will remain open questions until laboratory analyses can be conducted directly on these types of materials (Wray, 2013; Clark et al., 2021).

Additional knowledge would come from measurement of the isotopic compositions of individual dust grains and components adsorbed thereon. Analyses of the stable isotopes of hydrogen, carbon, and oxygen provide crucial information about the different volatile reservoirs on Mars, the extent of interaction between surface and atmosphere, hydrothermal interactions, temperatures and volatile loss (e.g., Shaheen et al., 2010). Examination of separate populations of grains should show the extent of different inputs. Measurement of the abundances of radiometric isotopes may also be possible, depending on attributes such as grain size, which could open the door to the eventual age-dating of individual grains.

\subsection{Study of carbon chemistry}

Martian dust may also be key to investigating the carbon chemistry of the martian surface. It is well understood that Mars is bathed in UV and cosmic radiation that are deleterious to preservation of organics. Photo-Fenton chemistry is one example of a process that can destroy organic compounds (Amat et al., 2004). Still, results from Curiosity have shown that organic carbon compounds are present in near-surface materials, although it is currently unclear whether these compounds originated on Mars or have been delivered by meteorites and micrometeorites (e.g., Freissinet et al., 2015; Eigenbrode et al., 2018; Franz et al., 2020). Also not known is how much of this organic material is transported and redistributed by dust. Indeed, fine-grained organic airfall dust associated with micrometeorites could be spread by atmospheric processes. This effect has been observed in the terrestrial record, with identification of extraterrestrial organic matter (from micrometeorites) distributed in a dust cloud in the $3.33 \mathrm{Ga}$ coastal volcanic sediments of the Barberton Greenstone Belt in South Africa (Gourier et al., 2019). On Mars, samples of airfall dust could provide a means for a survey of organic material at the surface of Mars.

\subsection{Corrosion}

Charged dust particles and the chemical entities they carry could contribute to corrosion of the mechanical parts of landed spacecraft and the degradation of electronics. Dust grain components also may catalyze undesirable chemistry with adverse effects for sensitive instrumentation. Knowing the bulk elemental composition of the dust is not sufficient to evaluate the potential to cause chemical corrosion of spacecraft surfaces. Only a complete characterization could reveal the presence and concentrations of hazardous corrosive enitites, such as free radicals, peroxides, and perchlorates (e.g., Turci et al., 2015; Chen et al., 2018; Wang et al., 2020, Encrenaz et al., 2012). Friction imparts a charge to the dust and thereby could increase its potential to catalyze surface chemistry (Wu et al., 2018). While there may be chemical issues specific to the geology of particular sites, airborne dust contains species that are guaranteed to be present for every spacecraft. Knowledge of those species would make it possible to design equipment resistant to them.
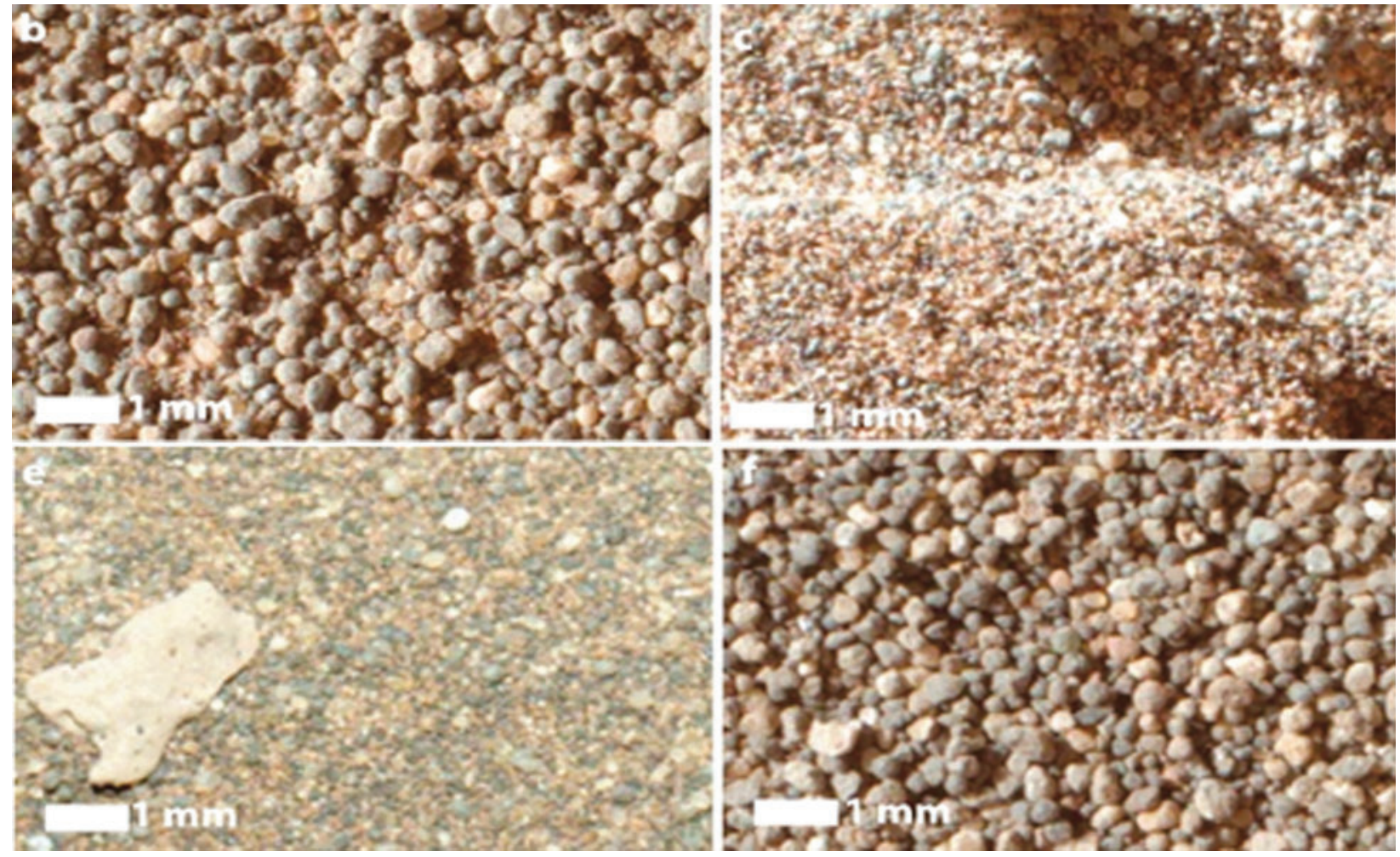

FIG. 3. Four samples of aeolian sand from the Bagnold Dune fields in Gale Crater, as imaged by the MAHLI instrument on Curiosity. The figure is part of Figure 3 in Ehlmann et al. (2017), in which a full description of the conditions under which the images were acquired can be found. 


\subsection{Importance of physical and magnetic properties}

A greater understanding of the physical properties of martian dust would inform the design of instrumentation with increasing complexity that could be delivered to Mars' surface. This includes information necessary for the safety of human exploration.

The size and shape distributions of dust particles can be particularly informative. Some properties have been inferred from the way that light is scattered or absorbed, or the temperature and wind speed needed to lift dust in a dust devil or dust storm (Newman et al., 2002; Ming and Morris, 2017). There have also been direct measurements of dust grains on the surface of Mars. Using instrumentation on the Curiosity rover, Ehlmann et al. (2017) carried out studies of grains from the Bagnold dunes in Gale Crater (Figure 3). The smallest grains they measured were $\sim 40 \mu \mathrm{m}$, but there were also likely to be grains below the resolution of the MAHLI instrument. The samples measured were part of an active dune field, that is, were partially consolidated rather than being recent airfall dust, which would be unconsolidated. Their position suggests that they are likely to be closer in properties to the regolith sample that would be collected by Perseverance rather than to airfall or airborne dust.

Thus, much more is expected to be learned from airfall dust samples, particularly of grains smaller than the resolution of rover instruments.

Knowing the physical and chemical properties of a large sampling of dust particles would make it possible to develop effective and targeted mitigation strategies for future human exploration. Furthermore, they are also significant factors in understanding how dust storms and dust devils begin and propagate and are measurements that can only be made in a laboratory on samples returned to Earth.

\subsection{Atmospheric dynamics and global circulation}

Knowing the abundance of aerosols and larger dust particles, particularly in the middle and upper atmosphere, is critical for spacecraft operations such as entry, descent and landing (EDL), take-off and ascent, and (for the upper atmosphere) orbital insertion. When dust (of any grain size) is present in the atmosphere, it absorbs sunlight, warming and causing expansion of, and altering the density profile of, the atmosphere. This particularly affects orbital insertion of spacecraft because it affects the position of atmospheric entry and the timing of parachute deployment. The dust that can be returned as samples would necessarily come from near ground level. However, it is this dust that moves into the middle and upper atmosphere via processes that are dependent on the properties of the available dust. Returned samples of nearsurface dust (both airborne and airfall, covering a range of particle sizes) would provide more accurate input parameters for models of the dynamics of the entire atmosphere.

Although some aspects of dust storm inception and propagation are understood (e.g., the largest storms occur near perihelion, in southern hemisphere summer), details such as the processes that control lifting and exchange of dust grains between the surface and atmosphere are unknown, although electrification processes related to atmospheric transport and suspension of dust are contributing factors (Harrison et al., 2016). Increasing our understanding of martian dust would likely allow us to better understand and predict martian dust storms. Complementary to the understanding of storms is the requirement to be able to mitigate damage caused by the dust to landed assets. Currently, the main method of cleaning the exterior surfaces of a lander or rover is to wait for wind gusts to sweep the dust away. The longevity of several martian rovers shows that this strategy is helpful but insufficient. Accordingly, greater knowledge of martian dust properties could help improve the design of some components as well as a more effective, reliable, and predictable recovery strategy.

FINDING D-1: An accumulation of airfall dust would be an unavoidable consequence of leaving M2020 sample tubes cached and exposed on the surface of Mars. Detailed laboratory analyses of this material would yield new knowledge concerning surface-atmosphere interactions that operate on a global scale, as well as provide input to planning for the future robotic and human exploration of Mars.

\subsection{Need for returned samples}

Many of the above investigations require instrumentation and measurement techniques only available in terrestrial laboratories (see, e.g., McLennan et al., 2012; Carrier et al., 2017; Beaty et al., 2019). For example, individual grains are sufficient for a wide range of measurements, including mineralogical variations, redox states, and isotopic measurements. These all require complex preparation procedures that cannot be carried out on the surface of Mars. Other studies require the analysis of large numbers of individual grains, numbers far more easily handled and processed in terrestrial laboratories. Analysis of a dust sample would act as a pathfinder for increasingly elaborate procedures, and as is the case for all returned samples, early analyses are likely to lead to discoveries and questions that require follow-up that can be done on other grains that have been returned. In addition, while there would be dust within the regolith samples to be collected, a sample of the dust that falls on bare tubes under current conditions, without the long-term interaction with the regolith, would provide a valuable baseline measurement of the dust provided to the regolith. One caveat regarding analysis of the dust collected from the external surfaces of the cached tubes is that these surfaces have not been cleaned to the same standards as the insides of the sample tubes. While this would not be significant for most measurements, it could affect the use of the samples for life detection-related studies.

FINDING D-2: The detailed information that is possible from analysis of airfall dust can only be obtained by investigation in Earth laboratories, and thus this is an important corollary aspect of MSR. The same information cannot be obtained from orbit, from in situ analyses, or from analyses of samples drilled from single locations. 


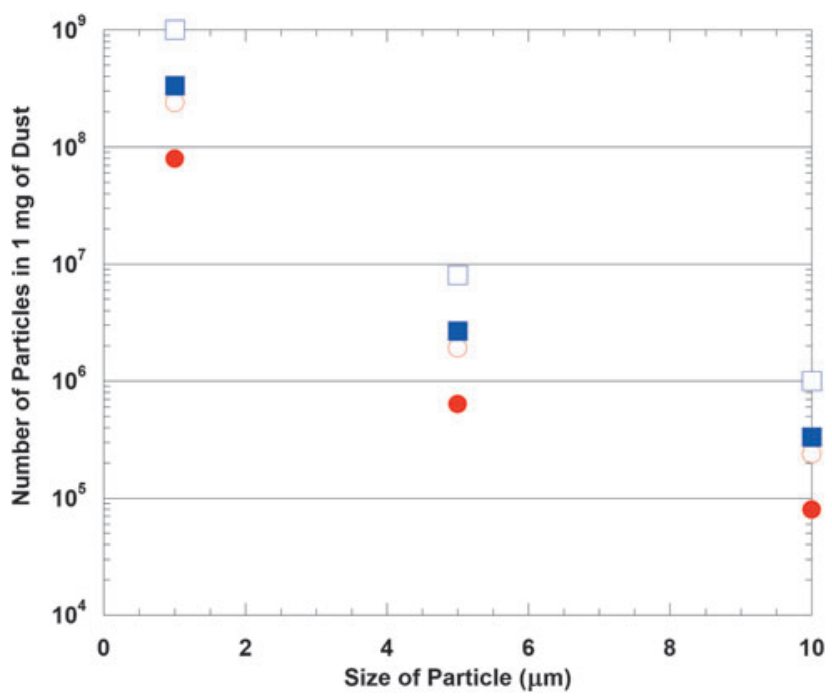

FIG. 4. Number of particles in $1 \mathrm{mg}$ of dust. Open symbols: assumed density of $1 \mathrm{~g} \mathrm{~cm}^{-3}$; filled symbols: assumed density of $3 \mathrm{~g} \mathrm{~cm}^{-3}$. Circles: spherical particle; Squares: cubic particle.

\section{How Much Dust Is Required?}

Studies of interplanetary dust grains and samples from the Stardust and Hayabusa missions have demonstrated that thorough characterization of micrometer-sized grains is possible using a variety of analytical techniques (AléonToppani et al., 2021). A $1 \mu \mathrm{m}$-sized grain is sufficiently large to enable determination of its morphology and texture, as well as measurement of its major and minor element chemistry, and even some isotopes. As shown in Figure 4, for realistic grain densities, a $1 \mathrm{mg}$ sample of martian dust would contain between $10^{6}$ to $10^{9}$ particles, depending on shape and size. It is clear that a $1 \mathrm{mg}$ sample of martian dust would provide sufficient material to characterize its bulk properties and inter-grain variability. Such studies would not only provide valuable scientific data but would be highly informative for more detailed study of the cached material, including information on the potential toxicity of the material. Contemporary, high resolution mass spectrometric techniques are sufficiently sensitive to identify both small and large organic molecules adhering to dust grains.

\subsection{How much airfall dust might we collect?}

In its current implementation, the M2020 mission plans to sample the regolith, with any admixed dust, using its waterfall drill bit. This is a specialized tool that scoops up material, then cascades it into a sample tube. Depending on discoveries made during traverses, samples may be taken from an aeolian deposit on the surface that has a very high concentration of airfall dust (e.g., similar to the RockNest sample taken by Curiosity). Finally, because the current implementation of MSR is associated with the caching of sample tubes on the martian surface for multiple years, the outsides of those tubes serve as a pathway to collect additional samples of airfall dust. However, it is likely that some fraction of this dust would be lost during retrieval of the tubes by SFR and their loading into the OS. In addition, the dust on the tubes would not be encapsulated; the dust from all of the tubes would be loose within the OS and vulnerable to mixing. Nevertheless, it would be an an extremely valuable addition to the inventory of material returned from Mars and, in this context, deserves scientific attention.

FINDING D-3: Given that at least some martian dust would be on the exterior surfaces of any sample tubes returned to Earth, the capability to receive and curate dust in an MSR Sample Receiving Facility (SRF) is essential.

No special equipment or technique is required to collect or store airfall dust. It is a sample of opportunity that would be present on the sample tubes. Indeed, it may be impossible to remove. We can approximate how much airfall dust might be obtained based on the following relatively restrictive assumptions:

Sample tube is $8 \mathrm{~cm}$ long (h) and $1 \mathrm{~cm}$ in diameter (2r); Dust is only on the side, and not the top or bottom; Dust covers $1 / 3$ of one side of the tube evenly to a depth of $10 \mu \mathrm{m}$ ( 2 monolayers of grains $5 \mu \mathrm{m}$ wide); Dust has a density of $1.5 \mathrm{~g} \mathrm{~cm}^{-3}$

Surface Area of $1 / 3$ of one side of a cylinder $=1 / 3 \pi \mathrm{rh}$ and mass $=$ volume $\times$ density

Volume of dust $=\frac{1}{3} \pi \mathrm{rh} \times$ depth of dust $=1 / 3 \pi \times 0.5 \times 8 \times$ $10 \times 10^{-4} \mathrm{~cm}^{-3}=4 \times 10^{-3} \mathrm{~cm}^{-3}$

Mass of dust $=4 \times 10^{-3} \times 1.5 \mathrm{~g}$

Mass of dust on one tube $=6 \mathrm{mg}$

If most of the tubes are similarly coated, then we could collect of the order of $\mathbf{1 0 0} \mathbf{~ m g}$ of airfall dust. This could be a minimum amount, given the density of the dust grains should be closer to $3 \mathrm{~g} \mathrm{~cm}^{-3}$, the dust coating more than 2 monolayers thick and more than a third of the tube length is coated. As well as coating the cached tubes, dust would almost certainly be present on, or in, several parts of the returned hardware, including the inside and the outside of the OS and the inside of the witness tubes. Although deliberate steps would be taken to minimize the dust on the outside of the OS for planetary protection reasons, it is unlikely that all of it would be removed.

\subsection{Airborne dust-a potential additional sampling opportunity}

There are strong arguments for going further than merely accepting the presence of dust on exterior surfaces. We can make a virtue of the serendipitous dust by recognizing the scientific and technical arguments for its retention and return. However, it would also be valuable to collect a sample of airborne dust directly from the atmosphere. A sample of the airborne dust (e.g., dust collected by drawing air through a filter) can be thought of as point sample of a larger and dynamic system. Interpreting such a sample would require knowledge of the sample's context in time and space. However, depending how it is done, its having a specific context significantly enhances is scientific value.

In contrast to the airfall dust, collection of airborne dust would require special collection and storage. Collection could be associated with the acquisition of a sample of atmosphere. A white paper (Jakosky et al., 2020) discussing a 


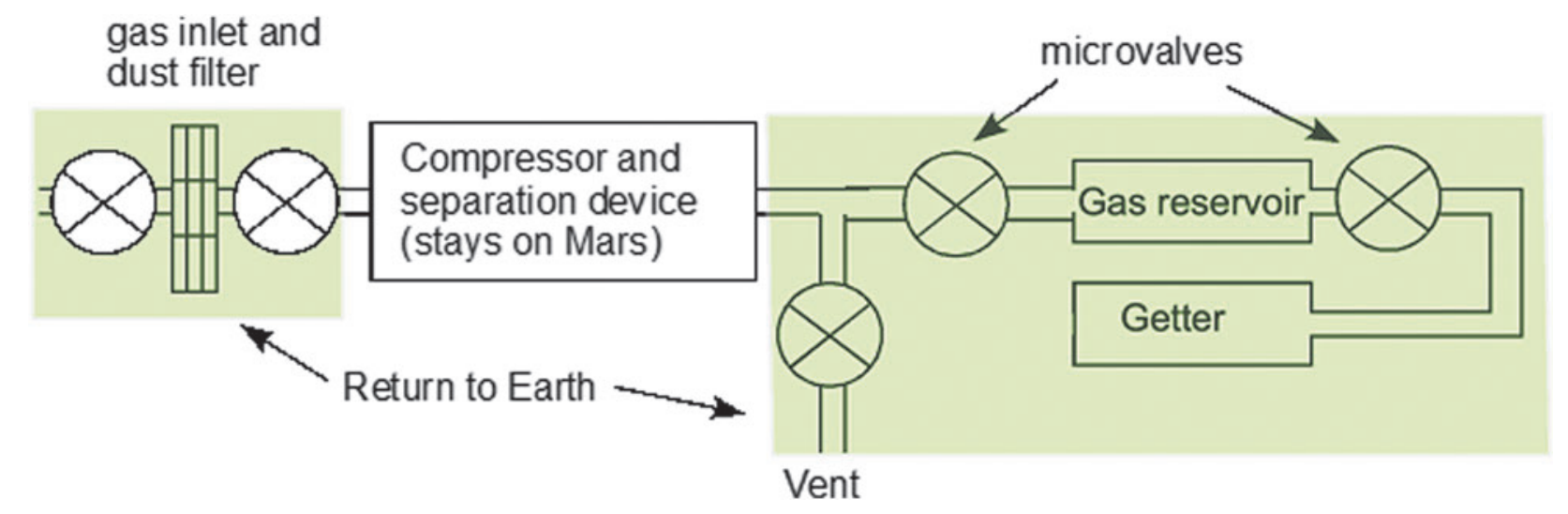

FIG. 5. Schematic of an assembly for collection of a sample of martian atmosphere that would also enable filtration of airborne dust. From Jakosky et al. (2020).

return of an atmospheric sample by the fetch rover has suggested the collection of a filtered sample of $\sim 100 \mathrm{~cm}^{3}$ with a pressure $\sim 100$ times ambient martian atmospheric pressure. This would require processing $10 \mathrm{~L}$ of martian atmosphere. Jakosky et al. (2020) included an extension to their proposed collector assembly that would enable filtration of the atmosphere as it was compressed (Figure 5).

The dust load of Mars' atmosphere close to the surface is variable, depending on weather conditions. Modeling has suggested that it might vary from about $2 \times 10^{-10} \mathrm{~g} \mathrm{~cm}^{-3}$ under hazy conditions up to $7 \times 10^{-8} \mathrm{~g} \mathrm{~cm}^{-3}$ in a dust devil (Metzger et al., 1999). Using the suggested turbo pump (manufactured by Creare, weighing $\sim 350 \mathrm{~g}$ with a flow rate of $1.21 \mathrm{~min}^{-1}$ ), we can calculate the following:

Flow rate of pump $=1.21 \mathrm{~min}^{-1}=1200 \mathrm{~cm}^{3} \mathrm{~min}^{-1}$ Dust load of the atmosphere $=2 \times 10^{-10} \mathrm{~g} \mathrm{~cm}^{-3}$ Dust collected $=2 \times 10^{-10} \times 1200 \mathrm{~g} \mathrm{~min}^{-1}$ $=240 \mathrm{ng} \mathrm{min}^{-1}$ $\approx 15 \mu \mathrm{g} \mathrm{h}^{-1}$

Pumping for 10 hours for 10 days would collect $\sim \mathbf{1} \mathbf{~ m g}$ of airborne dust under normal conditions. As calculated above, this would yield between $10^{6}$ and $10^{9}$ particles, depending on their size and density.

\section{Summary}

There are compelling scientific and operational arguments in favor of the collection both of airfall and airborne dust (collection of regolith dust is already envisaged).

- Dust analysis would provide critical information related to operations at Mars, not just for spacecraft operations, such as EDL and ascent, but also for understanding the effects of mechanical abrasion; corrosion due to the presence of chemical species, such as radicals; the reduction in sunlight for solar panel power sources; as well as chemical biohazards, including peroxides, that are toxic to humans.

- Dust measurements would provide key input to our Mars global circulation models.

- The physical, chemical, and spectroscopic characteristics of airfall and airborne dust would afford calibrations between direct and remote measurements and allow researchers to "ground truth" data acquired from orbiting and landed spacecraft. Combining spectroscopic, compositional and age data of dust grain populations would improve Mars chronologies.

- Detailed investigation of individual dust grains using in situ instruments is precluded by their micrometerlevel size.

- The capability to receive and curate dust is an essential requirement for the $\mathrm{SRF}$.

Collection of airfall dust is a scientific objective that does not reqire specialized equipment or storage. Adhered to the cached sample tubes, it would almost certainly be difficult to remove. Estimates of this serendipitous dust suggest that at least $100 \mathrm{mg}$ of material could be transferred into the OS. Retention of this dust affords a multitude of scientific opportunities for understanding the geological history of Jezero crater and Mars itself. Detailed characterization of this dust would also provide new knowledge that would be valuable for the design of future spacecraft and the safety of human spaceflight to Mars. Thus, we strongly recommend that dust on the exterior of the sample tubes is not actively removed before placing the tubes inside the OS.

SUMMARY STATEMENT: The fact that any sample tubes cached on the martian surface would accumulate some quantity of martian airfall dust presents a low-cost scientifically valuable opportunity. Some of this dust would inadvertently be knocked off as part of tube manipulation operations, but any dust possible should be loaded into the OS along with the sample tubes. This dust should be captured in an SRF and made available for detailed scientific analysis.

\section{Acknowledgments}

The decision to implement Mars Sample Return will not be finalized until NASA's completion of the National Environmental Policy Act (NEPA) process. This document is being made available for planning and information purposes only.

\section{Disclosure Statement}

No competing interests. 


\section{Funding Information}

A portion of this work was funded by the National Aeronautics and Space Administration (NASA), the European Space Agency (ESA), and the Canadian Space Agency (CSA).

A portion of this work was carried out at the Jet Propulsion Laboratory, California Institute of Technology, under a contract with the National Aeronautics and Space Administration (80NM0018D0004).

This work has partly (H. B.) been carried out within the framework of the NCCR PlanetS supported by the Swiss National Science Foundation. M.A.V's participation in MSPG2 was supported in part by a sabbatical leave-ofabsence from Michigan State University. M.-P.Z. was supported by projects PID2019-104205GB-C21 of Ministry of Science and Innovation and MDM-2017-0737 Unidad de Excelencia 'Maria de Maeztu'- Centro de Astrobiología (CSIC-INTA) (Spain).

\section{References}

Aleon-Toppani A, Brunetto R, Aleon J, et al. (2021) A preparation sequence for multi-analysis of $\mu \mathrm{m}$-sized extraterrestrial and geological samples. Meteorit Planet Sci 56:1151-1172.

Amat AM, Arques A, Miranda MA, et al. (2004) Photo-Fenton reaction for the abatement of commercial surfactants in a solar pilot plant. Sol Energy 77:559-566.

Beaty DW, Grady MM, McSween HY, et al. (2019) The potential science and engineering value of samples delivered to Earth by Mars sample return. Meteorit Planet Sci 54:S3-S152.

Berger JA, Schmidt ME, Gellert R, et al. (2016) A global Mars dust composition refined by the Alpha-Particle X-ray Spectrometer in Gale Crater. Geophys Res Lett 43:67-75.

Bertrand T, Wilson RJ, Kahre MA, et al. (2020) Simulation of the 2018 Global Dust Storm on Mars using the NASA Ames Mars GCM: A multitracer approach. J Geophys Res Planets 125.

Carrier BL, Beaty DW and Hecht MH (2017) The potential value of returning samples of martian dust and other granular materials for analysis in earth laboratories to preparing for the human exploration of Mars [abstract 6037]. In Dust in the Atmosphere of Mars and its Impact on Human Exploration, Houston.

Chen QC, Wang MM, Sun HY, et al. (2018) Enhanced health risks from exposure to environmentally persistent free radicals and the oxidative stress of PM2.5 from Asian dust storms in Erenhot, Zhangbei and Jinan, China. Environ Int 121:260-268.

Clark J, Sutter B, Archer PD, et al. (2021) A review of sample analysis at Mars-evolved gas analysis laboratory analog work supporting the presence of perchlorates and chlorates in Gale Crater, Mars. Minerals-Basel 11.

De Deckker P, Abed RMM, de Beer D, et al. (2008) Geochemical and microbiological fingerprinting of airborne dust that fell in Canberra, Australia, in October 2002. Geochem Geophys Geosyst 9:Q12Q10.

Edgett KS and Malin MC (2000) New views of Mars eolian activity, materials, and surface properties: Three vignettes from the Mars Global Surveyor Mars Orbiter Camera. $J$ Geophys Res Planets 105:1623-1650.

Ehlmann BL, Edgett KS, Sutter B, et al. (2017) Chemistry, mineralogy, and grain properties at Namib and High dunes, Bagnold Dune Field, Gale Crater, Mars: A synthesis of Curiosity rover observations. J Geophys Res Planets 122: 2510-2543.
Eigenbrode JL, Summons RE, Steele A, et al. (2018) Organic matter preserved in 3-billion-year-old mudstones at Gale Crater, Mars. Science 360:1096-1100.

Encrenaz T, Greathouse TK, Lefèvre F, et al. (2012) Hydrogen peroxide on Mars: Observations, interpretation and future plans. Planet Space Science 68:3-17.

Franz HB, Mahaffy PR, Webster CR, et al. (2020) Indigenous and exogenous organics and surface-atmosphere cycling inferred from carbon and oxygen isotopes at Gale crater. Nature Astronomy 4:526-532.

Freissinet C, Glavin DP, Mahaffy PR, et al. (2015) Organic molecules in the Sheepbed Mudstone, Gale Crater, Mars. J Geophys Res Planets 120:495-514.

Gourier D, Binet L, Calligaro T, et al. (2019) Extraterrestrial organic matter preserved in $3.33 \mathrm{Ga}$ sediments from Barberton, South Africa. Geochim et Cosmochim Acta 258:207-225.

Guzewich SD, Lemmon M, Smith CL, et al. (2019) Mars Science Laboratory observations of the 2018/Mars Year 34 Global Dust Storm. Geophys Res Lett 46:71-79.

Hanel RA, Conrath BJ, Schlachm B, et al. (1972) Infrared spectroscopy experiment on Mariner 9 Mission - preliminary results. Science 175:305.

Harrison RG, Barth E, Esposito F, et al. (2016) Applications of electrified dust and dust devil electrodynamics to martian atmospheric electricity. Space Sci Rev 203:299-345.

Hunt GR, Logan LM and Salisbury JW (1973) Mars: Components of infrared spectra and the composition of the dust cloud. Icarus 18:459-469.

Jakosky B, Amato M, Atreya S, et al. (2021) Scientific value of returning an atmospheric sample from Mars. Bulletin of the AAS 53.

Kahre MA, Hollingsworth JL, Haberle RM, et al. (2008) Investigations of the variability of dust particle sizes in the martian atmosphere using the NASA Ames General Circulation Model. Icarus 195:576-597.

Korotev RL, Zeigler RA and Floss C (2010) On the origin of impact glass in the Apollo 16 regolith. Geochim et Cosmochim Acta 74:7362-7388.

Kring DA (2006) Parameters of Lunar Soils. Lunar Exploration Initiative, Lunar and Planetary Institute and references therein. Presentation slides available online at https://www .lpi.usra.edu/science/kring/lunar_exploration/briefings/lunar_ soil_physical_properties.pdf.

Leovy CB (1973) Exchange of water vapor between atmosphere and surface of Mars. Icarus 18:120-125.

Leovy CB, Zurek RW and Pollack JB (1973) Mechanisms for Mars dust storms. J Atmos Sci 30:749-762.

Levine JS, Winterhalter D, Kerschmann R, et al. (2018) Mars atmospheric dust and human exploration. The Space Review, Feb. 5, 2018. Available online at http://www.thespacereview .com/article/3424/1.

Lorenz RD, Lemmon MT, Maki J, et al. (2020) Scientific observations with the InSight Solar Arrays: Dust, clouds, and eclipses on Mars. Earth and Space Science 7:e2019EA 000992

Mars 2020 SDT (2013) Committee members: Mustard, J.F (chair), M. Adler, A. Allwood, D.S. Bass, D.W. Beaty, J.F. Bell III, W.B. Brinckerhoff, M. Carr, D.J. Des Marais, B. Drake, K.S. Edgett, J. Eigenbrode, L.T. Elkins-Tanton, J.A. Grant, S. M. Milkovich, D. Ming, C. Moore, S. Murchie, T.C. Onstott, S.W. Ruff, M.A. Sephton, A. Steele, A. Treiman, 2013, Report of the Mars 2020 Science Definition Team available online at https://mepag.jpl.nasa.gov/reports/MEP/ Mars_2020_SDT_Report_Final.pdf. 
McLennan SM, Sephton MA, Allen C, et al. (2012) Planning for Mars returned sample science: Final report of the MSR end-to-end international science analysis group (E2E-iSAG). Astrobiology 12:175-230.

Metzger SM, Carr JR, Johnson JR, et al. (1999) Dust devil vortices seen by the Mars Pathfinder Camera. Geophys Res Lett 26:2781-2784.

Meyer MA, Kminek G, Beaty DW, et al. (2021) Final report of the MSR Science Planning Group 2 (MSPG2). Astrobiology 22(S1):S-5-S-26.

Ming DW and Morris RV (2017) Chemical, mineralogical and physical properties of martian dust and soil [abstract 6027]. In: Dust in the Atmosphere of Mars and Its Impact on Human Exploration, Houston.

Moeller RC, Jandura L, Rosette K, et al. (2021) The sampling and caching subsystem (SCS) for the scientific exploration of Jezero Crater by the Mars 2020 perseverance rover. Space Sci Rev 217.

Murray BC, Milton DJ, Cutts JA, et al. (1972) Geological framework of South Polar Region of Mars. Icarus 17:328.

Newman CE, Lewis SR, Read PL, et al. (2002) Modeling the martian dust cycle, 1 . Representations of dust transport processes. J Geophys Res Planets 107:6-1-6-18.

Olsen KS, Trokhimovskiy A, Montabone L, et al. (2021) Seasonal reappearance of $\mathrm{HCl}$ in the atmosphere of Mars during the Mars year 35 dusty season. Astron and Astroph 647:A161.

Reche I, D'Orta G, Mladenov N, et al. (2018) Deposition rates of viruses and bacteria above the atmospheric boundary layer. The ISME Journal 12:1154-1162.

Sagan C, Veverka J and Gierasch P (1971) Observational consequences of martian wind regimes. Icarus 15:253-278.

Shaheen R, Abramian A, Horn J, et al. (2010) Detection of oxygen isotopic anomaly in terrestrial atmospheric carbonates and its implications to Mars. Proc of the Nat Acad Sci 107:20213-20218.

Smith RJ, McLennan SM, Achilles CN, et al. (2021) X-Ray amorphous components in sedimentary rocks of Gale Crater, Mars: Evidence for ancient formation and long-lived aqueous activity. J Geophys Res Planets 126.

Sutter B, McAdam AC, Mahaffy PR, et al. (2017) Evolved gas analyses of sedimentary rocks and eolian sediment in Gale Crater, Mars: Results of the Curiosity rover's sample analysis at Mars instrument from Yellowknife Bay to the Namib Dune. J Geophys Res Planets 122:2574-2609.

Turci F, Corazzari I, Alberto G, et al. (2015) Free-radical chemistry as a means to evaluate lunar dust health hazard in view of future missions to the Moon. Astrobiology 15:371-380.

Wagner SA (2006) The Apollo Experience: Lessons Learned for Constellation Lunar Dust Management. NASA/TP-213726.
Wang A, Yan Y, Jolliff BL, et al. (2020) Chlorine release from common chlorides by martian dust activity. J Geophys Res Planets 125:e2019JE006283.

Wray JJ (2013) Gale Crater: The Mars Science Laboratory/ Curiosity Rover landing site. Int J Astrobiol 12:25-38.

Wu Z, Wang A, Farrell WM, et al. (2018) Forming perchlorates on Mars through plasma chemistry during dust events. Earth Planet Sci Lett 504:94-105.

Zellner NEB (2016). Lunar Regolith: Materials. In Encyclopedia of Lunar Science Ed. B Cudnik, Springer, pp 1-7.

Zellner NEB and Delano JW (2015) Ar-40/Ar-39 ages of lunar impact glasses: Relationships among Ar diffusivity, chemical composition, shape, and size. Geochim et Cosmochim Acta 161:203-218.

Zellner NEB, Delano JW, Swindle TD, et al. (2009) Evidence from Ar-40/Ar-39 ages of lunar impact glasses for an increase in the impact rate similar to $800 \mathrm{Ma}$ ago. Geochim et Cosmochim Acta 73:4590-4597.

For further information about MSPG2, please contact Michael Meyer (Michael.a.meyer@nasa.gov), Gerhard Kminek (Gerhard.kminek@esa.int), David Beaty (dwbeaty@jpl.nasa.gov), or Brandi Carrier (bcarrier@jpl.nasa.gov).

For further information on the technical content of this report, contact Monica Grady (monica.grady@open.ac.uk).

\begin{tabular}{|c|}
\hline $\begin{aligned} & \text { Acronyms Used } \\
& \text { BC }= \text { Basic Characterization } \\
& \text { ChemMin }= \text { Chemistry and Mineralogy Instrument } \\
& \text { EDL }= \text { Entry, Descent, and Landing } \\
& \text { iMOST }= \text { International MSR Objectives and Samples } \\
& \text { Team } \\
& \text { M2020 }= \text { Mars 2020; A NASA mission launched in July, } \\
& 2020 \text { and landed on Mars in Feb. 2021. The } \\
& \text { primary system is a sample-collecting rover } \\
& \text { named Perseverance. } \\
& \text { MAHLI }= \text { Mars Hand Lens Imager } \\
& \text { MAVEN }= \text { Mars Atmosphere and Volatile Evolution } \\
& \text { MGS }= \text { Mars Global Surveyor } \\
& \text { MSR }= \text { Mars Sample Return } \\
& \text { OS }= \text { Orbiting Sample Container } \\
& \text { SFR }= \text { Sample Fetch Rover } \\
& \text { SRF }= \text { Sample Receiving Facility } \\
& \text { TGO }= \text { ExoMars Trace Gas Orbiter } \\
& \text { XRD }= \text { X-Ray Diffractometer }\end{aligned}$ \\
\hline
\end{tabular}

